\title{
Screening Performance for Frailty Among Older Patients With Cancer: A Cross-Sectional Observational Study of Two Approaches
}

\author{
Toru Okuyama, MD, PhD ${ }^{\mathrm{a}, \mathrm{b}}$; Koji Sugano, MDª,b; Shinsuke Iida, MD, PhD ${ }^{\mathrm{d}}$; Takashi Ishida, MD, PhD ; \\ Shigeru Kusumoto, $\mathrm{MD}, \mathrm{PhD}^{\mathrm{d}}$; and Tatsuo Akechi, $\mathrm{MD}, \mathrm{PhD}^{\mathrm{a}, \mathrm{b}}$
}

\begin{abstract}
Objectives: To investigate the screening performance for frailty of the Vulnerable Elders Survey-13 (VES-13) and the 2-step approach consisting of the VES-13 plus the anhedonia (loss of interest or pleasure) item from Patient Health Questionnaire-9 (PHQ-9) among older patients with newly diagnosed cancer. Methods: This study involved 106 consecutive inpatients aged 65 years or older, newly diagnosed with malignant lymphoma or multiple myeloma, just before chemotherapy initiation. The participants were administered the VES-13, and also underwent a comprehensive geriatric assessment (CGA), including for depression and 6 other geriatric conditions, using validated measures, just before initiation of chemotherapy. We defined frailty as the presence of 2 or more geriatric conditions as determined by the CGA. Receiver operating characteristic analysis was performed. The 2-step screening approach was examined by post hoc analysis. Results: The average age of the subjects was 74 years. Among the 106 subjects, 50\% met the criteria for frailty. Using a cutoff score of 2 to 3 on the VES-13, a sensitivity and negative predictive value (NPV) of $72 \%$ of the screening tool was obtained for the condition of frailty. When the second step of the screening, consisting of the VES-13 plus anhedonia, was applied to the VES-13-negative patients, the sensitivity and NPV improved to $90 \%$ and $88 \%$, respectively. Conclusions: The 2-step approach exhibited better screening performance for frailty among patients with cancer than existing methods. Large prospective studies are required in the future to confirm this ability of the 2-step approach. (J Natl Comp Canc Netw 2015;13:1525-1531)
\end{abstract}

\section{Background}

The number of older patients (aged $\geq 65$ years) with cancer continues to increase worldwide, along with the growing size of the older population. ${ }^{1}$ Provision of optimal cancer treatment and supportive care for older patients with cancer is a challenging task for several reasons. Evidence of the effectiveness of anticancer treatments in older patients is insufficient, because few clinical trials enroll older patients with cancer. ${ }^{2}$ Furthermore, the older patient group is generally heterogeneous in terms of comorbidities; shows

From aivision of Psycho-Oncology and Palliative Care, Nagoya City University Hospital, Nagoya, Japan; ${ }^{b}$ Department of Psychiatry and Cognitive-Behavioral Medicine, Nagoya City University Graduate Schoo of Medical Sciences, Nagoya, Japan; 'Division of Respiratory Medicine, Juntendo University Faculty of Medicine and Graduate School of Medicine, Tokyo, Japan; and dDepartment of Hematology and Oncology, Nagoya City University Graduate School of Medical Sciences, Nagoya, Japan.

Submitted March 26, 2015; accepted for publication

September 30, 2015.

This work was supported in part by Grants-in-Aid for Cancer Research from the Ministry of Health, Labour and Welfare (grant number 201221008 decreased physical, psychological, and cognitive functions; lacks optimal decision-making capacity; and has poor social support. ${ }^{3}$ Physicians are often confronted with a difficult treatment decision regarding whether to use full-dose standard regimens, aimed at the best efficacy, or palliative regimens with greater priority assigned to safety. ${ }^{4}$

One of the modern approaches to overcoming this difficulty is to conduct a comprehensive geriatric assessment (CGA) to identify older patients with frailty before making treatment decisions. CGA is a multidi- 
Okuyama et al

mensional, often interdisciplinary, diagnostic process aimed at determining the medical, psychological, and functional capabilities of older persons in order to develop an overall plan for treatment and long-term follow-up. ${ }^{5}$ Recent research indicates the beneficial effect of CGA in predicting patients' tolerance to chemotherapy, treatment toxicity, treatment mortality, and survival, ${ }^{6-8}$ and in making better treatment choices. ${ }^{9-11}$ However, the implementation of CGA in actual oncology practice is often difficult because it requires investment of time and human resources.

The International Society of Geriatric Oncology $^{12}$ and the NCCN Clinical Practice Guidelines in Oncology (NCCN Guidelines) for Older Adult Oncology ${ }^{13}$ recommend screening older patients with cancer for frailty using screening tools (to view the most recent version of these NCCN Guidelines, visit NCCN.org). The Vulnerable Elders Survey-13 (VES-13) is a simple function-based tool, originally developed for the purpose of screening communitydwelling populations to identify older persons at risk for health deterioration. ${ }^{14}$ Some studies have indicated the usefulness of this scale to screen for frailty in mixed cancer populations. ${ }^{15,16}$ However, a recent systematic review revealed that none of the existing tools consistently yielded a sensitivity or negative predictive value (NPV) of $80 \%$ or greater in these patients, and that the VES-13 was no exception. ${ }^{12,17}$

The incidence of hematologic cancer, especially malignant lymphoma and multiple myeloma, has been increasing in older patients. ${ }^{18,19}$ The importance of CGA in these patients with cancer has been recognized, because the first-line treatment for most of these patients is chemotherapy, and the treatment outcomes in older patients with lymphoma/myeloma are poorer than those in younger patients. This difference cannot be attributed to the chronologic age difference alone but also to the clinical status of each patient, including cumulative decline in several physiological functions. ${ }^{4,20}$ To the best of our knowledge, no studies have been conducted to examine the usefulness of brief tools to screen for frailty among older patients with newly diagnosed hematologic malignancies as a separate group.

The purpose of this study was to investigate the screening ability of the VES-13 for frailty among older patients with newly diagnosed hematologic cancer. Also, we examined the usefulness of a 2-step screen- ing approach: conducting a second step of screening of VES-13-negative patients via post hoc analysis. We conducted this approach because we anticipated that the insufficient screening ability of theVES-13, reported previously, would be replicated in this study. ${ }^{17}$ We considered that the suboptimal screening ability of the VES-13 might be attributable to the heavy weighting given to the activities of daily living (ADL) in the questionnaire. Therefore, we examined 3 predetermined (not data-driven) secondstep screening items associated with depression, cognitive disturbance, or a combination of both. In addition, we explored whether the VES-13 and the 2-step screening method reflected the presence of each specific geriatric condition or not.

\section{Methods}

\section{Design}

This was a cross-sectional observation study.

\section{Procedures Overview}

All potentially eligible patients were identified upon admission to the hospital (consecutive sampling method) and assessed for eligibility before starting anticancer treatment. Patients were then asked to complete the VES-13 and the CGA described later.

\section{Setting/Sample}

This study was conducted in a tertiary care university hospital in Japan. The eligibility criteria for study inclusion were inpatients with newly diagnosed with malignant lymphoma or multiple myeloma, 65 years of age or older, informed of the cancer diagnosis, intending to undergo chemotherapy, and well enough to complete the survey questionnaire. Exclusion criteria included patients with severe mental or cognitive disorders diagnosed clinically, and/or the inability to understand the Japanese language.

This study was conducted with the approval of the Institutional Review Board and Ethics Committee of Nagoya City University Graduate School of Medical Sciences, Japan, and was conducted in accordance with the principles stated in the Declaration of Helsinki. Written consent was obtained from each eligible patient after a thorough explanation of the purpose and method of the study. When the participants could not fully understand the contents of the study protocol, both the patients' oral consent and the surrogates' written consent were obtained. 


\section{Measurements}

Vulnerable Elders Survey-13: The VES-13 is a 13-item self-administered instrument. ${ }^{14}$ The items pertain to the patients' age, self-rated overall health status, functional limitations in 5 domains of physical functioning, and 5 domains of functional disabilities. The original scoring method is described elsewhere. ${ }^{14}$ A score of 3 or greater has been shown to be predictive of functional decline and mortality in community-dwelling older. The Japanese version was developed using the forward-backward method of translation to Japanese, with the permission of the original developer.

Second-Step Screening \#1: Anhedonia: The item of anhedonia, or loss of pleasure or interest, was chosen for the second-step screening for 2 reasons: it is one of the 2 essential symptoms of depression and the single best item for depression screening ${ }^{21}$; it is also one of the initial symptoms of dementia. ${ }^{22}$ Anhedonia is included as one of the items in the Patient Health Questionnaire-9 (PHQ-9) instrument described later. ${ }^{23}$ The respondents were asked to rate the symptom on a 0 - to 3-point Likert scale (0: not at all; 3: nearly every day) according to the frequency noted over the previous 2 weeks. We defined the screening result as positive in cases for which the symptom frequency was rated as "2: present on more than half the days."

Second-Step Screening \#2: Delayed Recall: Delayed recall, included in the Mini-Mental State Examination (MMSE; described later), ${ }^{24}$ is known to be a good screening item for mild cognitive impairment and dementia. ${ }^{25} \mathrm{~A}$ tester asks the patients to memorize 3 objects, and then to recall them after the serial 7 subtraction test. The possible score ranges from 0 to 3; the higher the score, the better the cognition. We set the cutoff point at 2 to 3 .

Second-Step Screening \#3: Anhedonia and/or Delayed Recall: We also explored the usefulness of using the combination of anhedonia and delayed recall. $\mathrm{Pa}$ tients were considered as showing a positive screening result if they tested positive for at least 1 of the 2 items. Comprehensive Geriatric Assessment: The CGA consists of the following 7 domains assessed using reliable and validated tools. We defined frailty as the presence of 2 or more geriatric conditions on the CGA. ${ }^{17}$ The abnormal cutoff scores for each geriatric condition are summarized in Table 1. Psychiatrists in our research group conducted interviews, including the assessment of ADL, instrumental activities of daily life (IADLs), and cognitive function.

Activities of Daily Life: Patients' ADLs and mobility were assessed using the Barthel Index. ${ }^{26}$

Instrumental Activities of Daily Life: The Lawton IADL scale was used to assess IADLs. ${ }^{27}$

Comorbidity: The patients' comorbidities were scored using the Cumulative Illness Rating Scale for Geriatrics (CIRS-G) ${ }^{28}$ by attending physicians. Each comorbidity in a patient was assigned to 1 of 14 organ systems and rated from 0 (no comorbidity) to 4 (extremely severe comorbidity). We used the number of grade 3 (severe/constant significant disability/"uncontrollable" chronic problems) or 4 CIRS-G comorbidities (hematologic comorbidities were not investigated) for this study.

Nutrition: The body mass index was calculated to assess the nutritional status.

Polypharmacy: The number of medications that each subject was taking was investigated by a review of the medical records.

\begin{tabular}{|c|c|c|}
\hline Sample Characteristic & $\mathbf{N}$ & $(\%)$ \\
\hline \multicolumn{3}{|l|}{ Age (y) } \\
\hline \multicolumn{3}{|c|}{ Mean, $74.2 \pm 5.9($ range, $65-90) ;$ median, 74} \\
\hline \multicolumn{3}{|l|}{ Sex } \\
\hline Male & 56 & 53 \\
\hline \multicolumn{3}{|l|}{ Education } \\
\hline High school or higher & 61 & 58 \\
\hline \multicolumn{3}{|l|}{ Marital status } \\
\hline Married & 72 & 68 \\
\hline \multicolumn{3}{|l|}{ Household size } \\
\hline Living with someone & 89 & 84 \\
\hline \multicolumn{3}{|l|}{ Job } \\
\hline Employed (full-time/part-time) & 25 & 24 \\
\hline \multicolumn{3}{|l|}{ Diagnosis } \\
\hline Malignant lymphoma & 76 & 72 \\
\hline \multicolumn{3}{|l|}{ Ann Arbor staging } \\
\hline I & 13 & 17 \\
\hline II & 17 & 22 \\
\hline III & 20 & 26 \\
\hline IV & 26 & 34 \\
\hline International prognosis index,$\geq 3$ & 36 & 47 \\
\hline Multiple myeloma & 30 & 28 \\
\hline \multicolumn{3}{|l|}{ Durie and Salmon Staging } \\
\hline I & 1 & 3 \\
\hline II & 8 & 27 \\
\hline III & 21 & 70 \\
\hline \multicolumn{3}{|l|}{ ECOG performance status } \\
\hline 0 & 28 & 26 \\
\hline 1 & 47 & 44 \\
\hline 2 & 17 & 16 \\
\hline 3 & 12 & 11 \\
\hline 4 & 2 & 2 \\
\hline
\end{tabular}


Okuyama et al

Depression: Presence/absence of depression was assessed using the PHQ-9. ${ }^{23}$ The PHQ-9 consists of 9 items to evaluate the symptoms of major depressive disorder according to the criteria of the fourth edition of the Diagnostic and Statistical Manual of Mental Disorders (DSM-IV). The validity and reliability of the Japanese version of this scale has been established. ${ }^{29}$

Cognitive Function: The MMSE was used to evaluate the patients' cognitive function. ${ }^{24}$ The validity and reliability of the Japanese version of this scale has been established. ${ }^{30}$

\section{Statistical Methods}

Receiver operating characteristic (ROC) analysis was conducted to examine the screening performance of the VES-13 and the three 2-step approaches for patients with frailty defined by more than 2 deficits on the CGA. The optimal cutoff point was determined in order to minimize the sum of false-positive (1 - sensitivity) and false-negative (1 - specificity) test results. The area under the curve has been reported to be a useful measure to evaluate the screening performance. The value can range from 0 to 1.0; the greater the value, the better the screening performance. The positive predictive value (PPV) and NPV were also calculated to address the usefulness of the test in clinical practice. PPV is the probability that the condition is present given that the test is positive. NPV is the probability that the disease is absent given that the test is negative.

The 2-step screening approach was examined by post hoc analysis if both a sensitivity and NPV of $80 \%$ or greater of the VES-13 for the condition of frailty were not achieved. The 3 predetermined secondstep screenings described earlier were applied to patients with negative results from the VES-13. The combined results of the first and second screenings were then reported.

We also explored the screening performance of the VES-13 and the best screening method among three 2-step approaches for patients with each geriatric condition using the ROC analysis. This analysis allowed us to understand the specific geriatric domains that were strongly affected based on the results of each screening tool.

All of the statistical procedures were performed using the IBM SPSS software for Macintosh, version 21.0J.

\section{Results}

\section{Patient Characteristics}

A total of 149 potential participants were identified for the study. Of these, 25 patients were considered to be ineligible: 11 because of a serious physical condition, 8 because of cognitive disturbance, and 6 because of other reasons. Seven patients were excluded for logistic reasons. Eleven patients refused to provide consent for participation in the study. The sociodemographic and clinical characteristics of the remaining 106 patients are shown in Table 2. Of all the study subjects, $38 \%$ had diffuse large B-cell lymphoma, 34\% had other type of lymphoma, and $28 \%$ had multiple myeloma. These prevalences were almost consistent with those obtained from the population-based registry data in Japan, ${ }^{31}$ roughly indicating the representativeness of the study sample.

\section{Prevalence of Frailty}

The proportions of patients with 0 to 6 geriatric conditions were $25 \%, 25 \%, 11 \%, 15 \%, 16 \%, 7 \%$, and $1 \%$ in ascending order, respectively. Additionally, 50\% (95\% CI, 40-59) had more than 2 geriatric conditions, which was defined as frailty in this study.

\section{Screening for Frailty Using VES-13 and the} 2-Step Approach

The ROC analysis revealed that the optimal cutoff point score on the VES-13 was 2 to 3 , which was associated with $72 \%$ sensitivity and $79 \%$ specificity for the condition of frailty. Among patients with negative results from the VES-13, 19\%, 36\%, and 53\% had positive results on the second screening by anhedonia, delayed recall, or the combination of both, respectively. Among the 3 predetermined second-step screening approaches, the VES-13 plus anhedonia exhibited the best screening performance (Table 3).

\section{Screening for Specific Geriatric Conditions Using VES-13 and VES-13 Plus Anhedonia}

Table 1 summarizes the prevalence of each geriatric condition and the performance of the VES-13 alone and the VES-13 plus anhedonia for each specific geriatric condition. The VES-13 plus anhedonia showed a better sensitivity and NPV than VES-13 alone for not only depression but also most of the conditions included in the CGA. The mean sensitivity and NPV for the VES-13 alone and the VES13 plus anhedonia were $68 \%$ and $84 \%$, and $82 \%$ and $90 \%$, respectively. 
Screening Frailty Among Older Patients With Cancer

\begin{tabular}{|c|c|c|c|c|c|c|}
\hline Screening Method & $\begin{array}{l}\% \text { Positive } \\
\text { on Screening }\end{array}$ & AUC & $\begin{array}{l}\text { Sensitivity } \\
\text { (\%) }\end{array}$ & $\begin{array}{l}\text { Specificity } \\
(\%)\end{array}$ & $\begin{array}{l}\text { PPV } \\
\text { (\%) }\end{array}$ & $\begin{array}{l}\text { NPV } \\
\text { (\%) }\end{array}$ \\
\hline VES-13 alone & 48 & 0.85 & 72 & 79 & 79 & 72 \\
\hline \multicolumn{7}{|l|}{ 2-Step approach } \\
\hline VES-13 + anhedonia & 57 & 0.83 & 90 & 76 & 79 & 88 \\
\hline VES-13 + delayed recall & 66 & 0.62 & 78 & 46 & 59 & 68 \\
\hline $\begin{array}{l}\text { VES-13 + combination of } \\
\text { anhedonia and delayed } \\
\text { recall }\end{array}$ & 75 & 0.69 & 94 & 44 & 63 & 88 \\
\hline
\end{tabular}

Abbreviations: AUC, area under the curve; NPV, negative predictive value; PPV, positive predictive value; VES-13, Vulnerable Elders Survey-13.

\section{Discussion}

High sensitivity and high NPV are the most important desired characteristics of screening tools for frailty, because identification of all patients at risk for adverse outcomes is the highest priority. ${ }^{12}$ We found a suboptimal sensitivity of VES-13, consistent with previous reports; only 2 of the 11 studies reported so far, conducted in patients with solid cancer, described a sensitivity of greater than $80 \% .^{12}$

On the other hand, VES-13 plus anhedonia showed the best screening performance reported so far for existing tools, showing a fine balance between sufficient sensitivity (90\%) and NPV (88\%). These numbers indicated that, using this approach, approximately 9 in 10 frail patients may be expected to be correctly screened as frail, and fewer than 3 in 20 patients with negative screening results may be incorrectly identified as not frail. It has not recognized that increasing the number of domains assessed in a screening method is associated with improvement of screening ability. Thus, our result cannot be attributed simply to the addition of a non-performancebased domain on the VES-13, which is a functionbased tool.

Application of the anhedonia item can be rationalized in several ways. Screening tools should have sufficient sensitivity for the highly prevalent domains in CGA to achieve better screening performance for frailty. Depression is one such domain, and is also recognized to be associated with a poor outcome. ${ }^{3}$ However, the existing screening tools do not assign a high weight to depression. ${ }^{17}$ These results suggest the importance of including neuropsychiatric items in frailty screening tools. Anhedonia is 1 of 2 essential symptoms of depression, and therefore it enables more precise screening of patients with psychological problems than the VES-13 alone. Anhedonia is a

\begin{tabular}{|c|c|c|c|c|c|c|c|c|c|}
\hline Domain & $\begin{array}{l}\text { Instruments and } \\
\text { Abnormal Cutoff Score }\end{array}$ & $\begin{array}{l}\text { Prevalence of } \\
\text { Abnormality (\%) }\end{array}$ & $\begin{array}{l}95 \% \mathrm{Cl} \text { for } \\
\text { Prevalence }\end{array}$ & $\begin{array}{l}\text { Screening } \\
\text { Method }\end{array}$ & AUC & $\begin{array}{l}\text { Sensitivity } \\
(\%)\end{array}$ & $\begin{array}{l}\text { Specificity } \\
(\%)\end{array}$ & $\begin{array}{l}\text { PPV } \\
(\%)\end{array}$ & $\begin{array}{l}\text { NPV } \\
(\%)\end{array}$ \\
\hline \multirow[t]{2}{*}{$\overline{A D L}$} & \multirow[t]{2}{*}{ Barthel Index $\leq 90$} & \multirow[t]{2}{*}{33} & \multirow[t]{2}{*}{$24-42$} & VES-13 & 0.76 & 85 & 73 & 60 & 91 \\
\hline & & & & VES- $13+A$ & 0.78 & 94 & 62 & 55 & 96 \\
\hline \multirow[t]{2}{*}{ IADL } & Lawton Index, & \multirow[t]{2}{*}{45} & \multirow[t]{2}{*}{$35-55$} & VES-13 & 0.75 & 67 & 70 & 65 & 72 \\
\hline & $\begin{array}{l}\leq 7 \text { for female, } \\
\leq 4 \text { for male }\end{array}$ & & & VES-13+A & 0.73 & 83 & 64 & 66 & 82 \\
\hline \multirow[t]{2}{*}{ Comorbidity } & $\geq 1$ grade 3 or 4 & \multirow[t]{2}{*}{36} & \multirow[t]{2}{*}{$27-45$} & VES-13 & 0.70 & 66 & 64 & 51 & 77 \\
\hline & comorbidity on the CIRS-G & & & VES- $13+A$ & 0.67 & 79 & 55 & 60 & 82 \\
\hline \multirow[t]{2}{*}{ Nutrition } & Body mass index $\leq 18.5$ & \multirow[t]{2}{*}{11} & \multirow[t]{2}{*}{$5-17$} & VES-13 & 0.58 & 58 & 55 & 14 & 91 \\
\hline & $\mathrm{kg} / \mathrm{m}^{2}$ & & & VES- $13+A$ & 0.51 & 58 & 44 & 12 & 89 \\
\hline \multirow[t]{2}{*}{ Medications } & \multirow[t]{2}{*}{ Taking $\geq 5$ medications } & \multirow[t]{2}{*}{17} & \multirow[t]{2}{*}{$10-24$} & VES-13 & 0.71 & 67 & 58 & 25 & 90 \\
\hline & & & & VES- $13+A$ & 0.59 & 72 & 47 & 22 & 89 \\
\hline \multirow[t]{2}{*}{ Depression } & \multirow{2}{*}{$\begin{array}{l}\geq 2 \text { depressive symptoms, } \\
\text { with at least one of } \\
\text { the symptoms being } \\
\text { depressed mood or } \\
\text { anhedonia on the PHQ-9 }\end{array}$} & \multirow[t]{2}{*}{30} & \multirow[t]{2}{*}{$21-39$} & VES-13 & 0.69 & 66 & 62 & 43 & 81 \\
\hline & & & & VES-13+A & 0.79 & 97 & 61 & 52 & 98 \\
\hline \multirow{2}{*}{$\begin{array}{l}\text { Cognitive } \\
\text { dysfunction }\end{array}$} & \multirow[t]{2}{*}{ MMSE score $\leq 23$} & \multirow[t]{2}{*}{23} & \multirow[t]{2}{*}{$15-31$} & VES-13 & 0.70 & 71 & 61 & 35 & 88 \\
\hline & & & & VES- $13+A$ & 0.70 & 88 & 52 & 35 & 94 \\
\hline
\end{tabular}

Abbreviations: ADL, activities of daily living; AUC, area under the curve; CIRS-G, Cumulative Illness Rating Scale for Geriatrics; IADL, instrumental activities of daily living; MMSE, Mini-Mental State Examination; NPV, negative predictive value; PHQ-9, Patient Health Questionnaire-9; PPV, positive predictive value; ROC, receiver operating characteristic; VES-13, Vulnerable Elders Survey-13; VES-13+A, Vulnerable Elders Survey-13 plus the anhedonia item from the PHQ-9. 
Okuyama et al

less stigmatized item than depressive mood, which is the other essential symptom of depression. Furthermore, anhedonia is common and closely associated with cognitive dysfunction. ${ }^{32,33}$ As we expected, use of the VES-13 plus anhedonia tool yielded a better sensitivity and NPV for the detection of depression and cognitive dysfunction. Interestingly, it also resulted in a better sensitivity and NPV for various conditions, such as IADLs and comorbidity, compared with the sensitivity and NPV of the VES-13 alone. Anhedonia may be associated with other geriatric conditions than depression, although the causality remains unknown.

Several issues regarding the study design may limit our confidence in the findings of this study. First, the criterion for frailty applied in this study was arbitrary. However, no gold standard has been established, and many existing studies apply the number of geriatric conditions as a definition of frailty. ${ }^{17}$ Secondly, the applicability of the study results to the general population of older patients with cancer may not be justifiable, considering the sample characteristics of this study. Third, the performance of the 2-step approach was examined using post hoc analysis. In addition, the fact that this study was conducted at a single institution in Japan may have introduced institution bias.

Alternately, this study also had several strengths. Patients were consecutively recruited, and the rejection rate was minimal. Geriatric assessments were conducted comprehensively using internationally validated measures. To the best of our knowledge, this study is the first to conduct screening of older patients with newly diagnosed hematologic cancer for frailty. In addition, this study has established the VES-13 as the first validated frailty screening tool for Japanese patients with cancer.

We found a high prevalence of frailty in older patients with cancer before they were started on anticancer treatment, indicating the importance of better understanding and identifying this problem. Although the beneficial effect of CGA in predicting various outcomes has been recognized, implementation of CGA in actual oncology practice is often difficult. Use of the VES-13 plus anhedonia tool in older patients with cancer before initiating anticancer treatment will facilitate the implementation of CGA through enabling the medical staff to identify patients who would gain the most benefit from this evaluation, thereby enabling the most efficient use of medical resources.

\section{Conclusions}

The 2-step approach of administering the VES-13 plus anhedonia showed better performance in screening for frailty among patients with cancer compared with existing methods, indicating the potential clinical usefulness of this method. Future large prospective studies are required to confirm this screening performance in various settings. Furthermore, research is required to clarify that implementation of frailty screening would result in the improvement of care and treatment, and thereby better patient outcomes.

\section{Acknowledgments}

The authors wish to express their gratitude to Dr. Debra Saliba for granting permission for the development of the Japanese version of the VES-13. They would also like to acknowledge the contribution and commitment to this study of Tomohiro Nakaguchi, MD; Yosuke Kubota, MD; Yosinori Ito, BA; and Sayo Aiki, MD.

\section{References}

1. Howlader N, Noone AM, Krapcho M, et al, eds. SEER Cancer Statistics Review, 1975-2011. National Cancer Institute, Bethesda, MD. Available at: http://seer.cancer.gov/csr/1975_2011/, based on November 2013 SEER data submission, posted to the SEER web site, April 2014. 2014. Accessed November 11, 2015.

2. Bellera C, Praud D, Petit-Moneger A, et al. Barriers to inclusion of older adults in randomised controlled clinical trials on Non-Hodgkin's lymphoma: a systematic review. Cancer Treat Rev 2013;39:812-817.

3. Extermann M, Hurria A. Comprehensive geriatric assessment for older patients with cancer. J Clin Oncol 2007;25:1824-1831.

4. Peyrade F, Gastaud L, Ré D, et al. Treatment decisions for elderly patients with haematological malignancies: a dilemma. Lancet Oncol 2012;13:e344-352.

5. Repetto L, Venturino A, Fratino L, et al. Geriatric oncology. Eur J Cancer 2003;39:870-880.

6. Ramjaun A, Nassif MO, Krotneva S, et al. Improved targeting of cancer care for older patients: a systematic review of the utility of comprehensive geriatric assessment. J Geriatr Oncol 2013;4:271-281.

7. Winkelmann N, Petersen I, Kiehntopf M, et al. Results of comprehensive geriatric assessment effect survival in patients with malignant lymphoma. J Cancer Res Clin Oncol 2011;137:733-738.

8. Puts MT, Santos B, Hardt J, et al. An update on a systematic review of the use of geriatric assessment for older adults in oncology. Ann Oncol 2014;25:307-315.

9. Caillet P, Canoui-Poitrine F, Vouriot J, et al. Comprehensive geriatric assessment in the decision-making process in elderly patients with cancer: ELCAPA study. J Clin Oncol 2011;29:3636-3642.

10. Tucci A, Ferrari S, Bottelli C, et al. A comprehensive geriatric assessment is more effective than clinical judgment to identify elderly diffuse large cell lymphoma patients who benefit from aggressive therapy. Cancer 2009; 115:4547-4553 
11. Olivieri A, Gini G, Bocci C, et al. Tailored therapy in an unselected population of 91 elderly patients with DLBCL prospectively evaluated using a simplified CGA. Oncologist 2012;17:663-672.

12. Decoster L, Van Puyvelde $K$, Mohile S, et al. Screening tools for multidimensional health problems warranting a geriatric assessment in older cancer patients: an update on SIOG recommendations. Ann Oncol 2015;26:288-300.

13. Hurria A, Wildes T, Baumgartner J, et al. NCCN Clinical Practice Guidelines in Oncology: Older Adult Oncology. Version 2.2015. Available at: NCCN.org. Accessed November 9, 2015.

14. Saliba D, Elliott M, Rubenstein LZ, et al. The Vulnerable Elders Survey: a tool for identifying vulnerable older people in the community. J Am Geriatr Soc 2001;49:1691-1699.

15. Mohile SG, Bylow K, Dale W, et al. A pilot study of the vulnerable elders survey-13 compared with the comprehensive geriatric assessment for identifying disability in older patients with prostate cancer who receive androgen ablation. Cancer 2007;109:802-810.

16. Luciani A, Ascione G, Bertuzzi C, et al. Detecting disabilities in older patients with cancer: comparison between comprehensive geriatric assessment and vulnerable elders survey-13. J Clin Oncol 2010;28:20462050.

17. Hamaker ME, Jonker JM, de Rooij SE, et al. Frailty screening methods for predicting outcome of a comprehensive geriatric assessment in elderly patients with cancer: a systematic review. Lancet Oncol 2012;13:e437-444.

18. Thieblemont C, Coiffier B. Lymphoma in older patients. J Clin Oncol 2007;25:1916-1923.

19. Palumbo A, Bringhen S, Ludwig $\mathrm{H}$, et al. Personalized therapy in multiple myeloma according to patient age and vulnerability: a report of the European Myeloma Network (EMN). Blood 2011;118:4519-4529.

20. Ludwig H, Durie BG, Bolejack V, et al. Myeloma in patients younger than age 50 years presents with more favorable features and shows better survival: an analysis of 10549 patients from the International Myeloma Working Group. Blood 2008;111:4039-4047.

21. Mitchell AJ. Are one or two simple questions sufficient to detect depression in cancer and palliative care? A Bayesian meta-analysis. $\mathrm{Br} \mathrm{J}$ Cancer 2008;98:1934-1943.
22. Mossaheb N, Zehetmayer S, Jungwirth S, et al. Are specific symptoms of depression predictive of Alzheimer's dementia? J Clin Psychiatry 2012;73:1009-1015.

23. Spitzer RL, Kroenke K, Williams JB. Validation and utility of a selfreport version of PRIME-MD: the PHQ primary care study. Primary Care Evaluation of Mental Disorders. Patient Health Questionnaire. JAMA 1999;282:1737-1744.

24. Folstein MF, Folstein SE, McHugh PR. "Mini-mental state". A practical method for grading the cognitive state of patients for the clinician. J Psychiatr Res 1975;12:189-198.

25. Loewenstein DA, Barker WW, Harwood DG, et al. Utility of a modified Mini-Mental State Examination with extended delayed recall in screening for mild cognitive impairment and dementia among community dwelling elders. Int J Geriatr Psychiatry 2000;15:434-440.

26. Mahoney FI, Barthel DW. Functional evaluation: the Barthel Index. Md State Med J 1965;14:61-65.

27. Lawton MP, Brody EM. Assessment of older people: self-maintaining and instrumental activities of daily living. Gerontologist 1969;9:179-186.

28. Miller MD, Paradis CF, Houck PR, et al. Rating chronic medical illness burden in geropsychiatric practice and research: application of the Cumulative Illness Rating Scale. Psychiatry Res 1992;41:237-248.

29. Muramatsu K, Miyaoka H, Kamijima K, et al. The patient health questionnaire, Japanese version: validity according to the miniinternational neuropsychiatric interview-plus. Psychol Rep 2007;101(3 Pt 1):952-960.

30. Ideno Y, Takayama M, Hayashi K, et al. Evaluation of a Japanese version of the Mini-Mental State Examination in elderly persons. Geriatr Gerontol Int 2012;12:310-316.

31. Chihara D, Ito H, Matsuda T, et al. Differences in incidence and trends of haematological malignancies in Japan and the United States. Br J Haematol 2014;164:536-545.

32. Lopez OL, Becker JT, Sweet RA, et al. Psychiatric symptoms vary with the severity of dementia in probable Alzheimer's disease. J Neuropsychiatry Clin Neurosci 2003;1:346-353.

33. Mackin RS, Insel P, Aisen PS, et al; on behalf of the Alzheimer's Disease Neuroimaging Initiative. Longitudinal stability of subsyndromal symptoms of depression in individuals with mild cognitive impairment: relationship to conversion to dementia after 3 years. Int J Geriatr Psychiatry 2012;27:355-363. 